\title{
Applications of Some Generalized Janowski Meromorphic Multivalent Functions
}

\author{
Bakhtiar Ahmad (D), Muhammad Ghaffar Khan ${ }^{1},{ }^{2}$ Maslina Darus $\left(\mathbb{D},{ }^{3}\right.$ \\ Wali Khan Mashwani $\mathbb{D}^{2}$, and Muhammad Arif ${ }^{1}$ \\ ${ }^{1}$ Department of Mathematics, Abdul Wali Khan University Mardan, Mardan 23200, Pakistan \\ ${ }^{2}$ Institute of Numerical Sciences, Kohat University of Science and Technology, Kohat 26000, Pakistan \\ ${ }^{3}$ Department of Mathematical Sciences, Faculty of Science and Technology, Universiti Kebangsaan Malaysia, Bangi, \\ Selangor 43600, Malaysia
}

Correspondence should be addressed to Muhammad Ghaffar Khan; ghaffarkhan020@gmail.com

Received 11 November 2020; Revised 2 July 2021; Accepted 15 July 2021; Published 26 July 2021

Academic Editor: Ming-Sheng Liu

Copyright () 2021 Bakhtiar Ahmad et al. This is an open access article distributed under the Creative Commons Attribution License, which permits unrestricted use, distribution, and reproduction in any medium, provided the original work is properly cited.

\begin{abstract}
In this article, the ideas of post-quantum calculus and meromorphic multivalent functions are combined and some applications of these functions are discussed. We introduce a new subclass of meromorphic multivalent functions in association with Janowski domain. We investigate and study some useful geometric properties of this class of functions such as sufficiency criteria, distortion problem, growth theorem, radii of starlikeness and convexity, convex combination, and coefficient estimates for this class.
\end{abstract}

\section{Introduction and Definitions}

In this article, we introduce a new subclass of meromorphic multivalent functions in parameter $\alpha$, in post-quantum analogue. The quantum calculus ( $q$-calculus) is the generalization of classical calculus by replacing the notion of limits with a parameter $q$. In the field of geometric function theory (GFT), the $q$-generalization of different classes of analytic and meromorphic functions is the current focus of various prominent researchers. Many generalizations have been made and various properties are discussed for these generalizations. This motivation in the recent past is due to its numerous mathematical and physical applications. Jackson started this area of mathematics with the generalization of derivative and integral in $q$-analogue, which are known as $q$-derivative and $q$-integral $[1,2]$. The latest advancements in this field can be traced to Srivastava, who along with Bansal [3], investigated a certain family of $q$-Mittag-Leffer functions. Aldawish and Darus [4] gave results on starlikeness of $q$-generalized functions. Some results such as coefficient estimates for $q$-starlike and $q$-convex functions were evaluated by Seody and Aouf [5]. Similar results for some other subclasses of $q$-starlike functions were evaluated by Fan et al. in [6]. Mahmood and Sokół in [7] introduced a class in conical domain associated with Ruscheweyh $q$-differential operator and investigated its various properties. Shi et al. [8] used a generalized operator in the introduction and study of a class of analytic functions. Wang et al. [9] gave a useful generalization of Choi-Saigo-Srivastava operator in $q$-analogue. For other interesting results, the reader is referred to the work published in $[10,11]$. Similarly, the trend was carried to meromorphic functions by various researchers. Ahmad et al. [12] gave the investigation of $q$-analogue of meromorphic multivalent functions in lemniscate of Bernoulli domain and obtained some interesting results. Arif and Ahmad [13] introduced and studied $q$-analogue of a meromorphic multivalent operator and presented some interested results. The convex generalization of meromorphic multivalent functions in $q$-analogue can be seen in [14]. Further work by Ahmad and Arif [15] generalized a subclass of meromorphic multivalent close to convex functions via a $q$-operator. 
We extend the idea from $q$-calculus to post-quantum calculus $((P, Q)$-calculus) for the meromorphic multivalent functions. In $(P, Q)$-calculus, there is $(P, Q)$-number with two independent variables $P$ and $Q$. This was first considered by Jagannathan and Chakrabarti [16]. They defined the $(P, Q)$-number and generalized several forms of $q$-oscillator algebras, which are known in the field of physics. In the $(P, Q)$-analogue of mathematical concepts, the work of White and Wachs [17] is of most importance. They obtain the generating function of the joint distribution of pairs of statistics known as $(P, Q)$-Stirling number. In the field of GFT, the recent research work on generalizing various classes in $(P, Q)$-analogue can be seen in $[18,19]$. Hence, various applied and theoretical problems have led the way to the creation of $(P, Q)$-calculus. Since 1991, many researchers in the fields of mathematics and physics have contributed to the development of $(P, Q)$-calculus.

Motivated from the above discussed work, in which many ideas have been generalized to post-quantum analogue, we introduce a new class of meromorphic multivalent functions associated with Janowski domain. To better understand this class, we characterize with some results relating to bounds of its coefficients in its power series. Furthermore, we give well-known results of distortion and growth for these functions. In the next section, the region in the open unit disc is evaluated where these functions would behave like starlike and convex functions. After that, an important result of convex combinations is presented. In Section 7, we discuss conclusion and potential future work of this research.

We give few basic concepts of $(P, Q)$-calculus in the following which are closely related to our work.

Any $(P, Q)$-number can be defined mathematically as

$$
\begin{aligned}
{[n]_{P, Q} } & :=\frac{P^{n}-Q^{n}}{P-Q} \\
& =P^{n-1}+P^{n-1} Q+P^{n-1} Q+\cdots+Q^{n-1},
\end{aligned}
$$

where $0<Q<P \leq 1$ and $P \neq Q$.

To make things a bit simpler, we will use the following notation throughout this article:

$$
F_{n}=[n]_{P, Q} \cdot
$$

Some elementary properties of the $(P, Q)$-numbers are discussed in the following lines and can be verified easily:

(i) $F_{n_{1}+n_{2}}=Q^{n_{2}} F_{n_{1}}+P^{n_{1}} F_{n_{2}}=P^{n_{2}} F_{n_{1}}+Q^{n_{1}} F_{n_{2}}$.

(ii) $F_{n}=(P+Q) F_{n-1}+P Q F_{n-2}$.

(iii) The $(P, Q)$-factorial is defined by

$$
F_{n} !=F_{n} F_{n-1} F_{n-2}, \ldots, F_{1}
$$

for $n \geq 1$ while $F_{0} !=1$.

(iv) Similarly, $(P, Q)$-derivative is defined mathematically as

$$
\mathscr{D}_{P, Q} f(z)=\frac{f(P z)-f(Q z)}{(P-Q) z},
$$

with $z \neq 0$ and $P \neq Q$.

(v) With simple calculations, one can easily deduce the following identity:

$$
\mathscr{D}_{P, Q} z^{n}=F_{n} z^{n-1} \text {. }
$$

For the special value, when $P=1$, this derivative becomes the well-known $q$-derivative in $q$-calculus.

In this article, we introduce a new class $\mathscr{M}_{P, Q}(p, \alpha, L, M)$. The class $\mathscr{M}_{P, Q}(p, \alpha, L, M)$ is a subclass of meromorphic multivalent functions in $(P, Q)$-analogue in Janowski domain which generalizes some important classes both in classical and in $q$-analogue of this field. Then, we investigate some important properties of this class $\mathscr{M}_{P, Q}(p, \alpha, L, M)$.

Let $\mathfrak{A}_{p}$ denote the class of all meromorphic multivalent functions $f$ that are analytic in the punctured disc $\mathbb{D}=\{z \in \mathbb{C}: 0<|z|<1\}$, and it is represented by

$$
f(z)=\frac{1}{z^{p}}+\sum_{k=1}^{\infty} a_{k+p} z^{k+p}, \quad z \in \mathbb{D} .
$$

Next, we recall the definition of subordination, and for two functions $h_{1}, h_{2} \in \mathscr{A}$, we say $h_{1}$ is subordinated to $h_{2}$ and is symbolically written as

$$
h_{1} \prec h_{2},
$$

if there exist an analytic function $w(z)$ with the property

$$
\begin{gathered}
|w(z)| \leq|z|, \\
w(0)=0,
\end{gathered}
$$

such that

$$
h_{1}(z)=h_{2}(w(z))
$$

Furthermore, if $h_{2} \in \mathcal{S}$, then the above condition becomes

$$
\begin{aligned}
h_{1} \prec h_{2} \Leftrightarrow h_{1}(0) & =h_{2}(0), \\
h_{1}(\mathbb{D}) & =h_{2}(\mathbb{D}) .
\end{aligned}
$$

Definition 1. Let $-1 \leq M<L \leq 1, \alpha \geq 0,0<P, Q \leq 1$, and $P \neq Q$. Then, the function $f \in \mathfrak{A}_{p}$ will be in the class $\mathscr{M}_{P, Q}(p, \alpha, L, M)$ if it satisfies the condition 


$$
\frac{-1}{1-\alpha}\left(\frac{P^{p} Q^{p} z \mathscr{D}_{P, \mathrm{Q}} f(z)}{F_{p} f(z)}+\alpha \frac{P^{2 p+1} Q^{2 p+1} z^{2} \mathscr{D}_{P, Q}^{2} f(z)}{F_{p} F_{p+1} f(z)}\right)<\frac{1+L z}{1+M z} .
$$

Equivalently, a function $f(z) \in \mathfrak{A}_{p}$ will be in the class $\mathscr{M}_{P, Q}(p, \alpha, L, M)$ if and only if the following inequality holds true

$$
\left|\frac{\left(P^{p} Q^{p} z \mathscr{D}_{P, Q} f(z) / F_{p} f(z)\right)+\alpha\left(P^{2 p+1} Q^{2 p+1} z^{2} \mathscr{D}_{P, \mathrm{Q}}^{2} f(z) / F_{p} F_{p+1} f(z)\right)+1-\alpha}{(1-\alpha) L+M\left(\left(P^{p} Q^{p} z \mathscr{D}_{P, \mathrm{Q}} f(z) / F_{p} f(z)\right)+\alpha\left(P^{2 p+1} Q^{2 p+1} z^{2} \mathscr{D}_{P, \mathrm{Q}}^{2} f(z) / F_{p} F_{p+1} f(z)\right)\right)}\right|<1 .
$$

We note that, for various specific values of $L, M, Q, P$, and $\alpha$, we get the following well-known subclasses.

(1) If $\alpha=0, P=1$, and $p=1$, we get the class of meromorphic $q$-starlike functions associated with Janowski functions studied by Mahmood et al. [20]

(2) For $P=1, Q \longrightarrow 1^{-}$, and $p=1$, our class generalizes the class of meromorphic $\alpha$-convex functions introduced by Ali and Ravichandran [21]

(3) Putting $L=1, \alpha=0, P=1, Q \longrightarrow 1^{-}$, and $M=-1$, we get $\mathscr{M} \delta_{p}^{*}$, the class of meromorphic $p$-valent starlike functions investigated by Royster [22]

(4) For $\alpha=0, P=1, Q \longrightarrow 1^{-}, L=1, M=-1, \quad$ and $p=1$, we get the class of meromorphic starlike functions studied by Pommerenke [23]

For our main results, we will need the following lemma

Lemma 1 (see [24]). For a function $h(z)$, if analytic in $D$ and is represented by the following,

$$
h(z)=1+\sum_{n=1}^{\infty} c_{n} z^{n}
$$

and $k(z)$ is analytic and convex in $D$ with

$$
k(z)=1+\sum_{n=1}^{\infty} k_{n} z^{n}
$$

and $h(z)<k(z)$, then

$$
\left|c_{n}\right| \leq\left|k_{1}\right|
$$

where $n \in N$.

Now, we give some properties for class $\mathscr{M}_{P, Q}(p, \alpha, L, M)$.

\section{Sufficiency Criteria}

In this section, we derive the condition under which functions will belong to this class $\mathscr{M}_{P, Q}(p, \alpha, L, M)$.

Theorem 1. If $f$ belongs to $\mathfrak{A}_{p}$, represented by (6), then the function $f$ will be in $\mathscr{M}_{P, Q}(p, \alpha, L, M)$ if and only if the following inequality holds:

$$
\begin{aligned}
& \sum_{n=1}^{\infty}\left((1+L)(1-\alpha) F_{p} F_{p+1}+(1+M)\left(P^{p} Q^{p} F_{p+1} F_{p+n}+\alpha P^{2 p+1} Q^{2 p+1} F_{p+n} F_{p+n-1}\right)\right)\left|a_{n+p}\right| \\
& \leq(L-M)(1-\alpha) F_{p} F_{p+1} .
\end{aligned}
$$


Proof. Let us suppose that the first inequality (16) holds. Then, to show that $f$ is in the class $\mathscr{M}_{P, Q}(p, \alpha, L, M)$, we will prove inequality (12). For this, consider

$$
\begin{aligned}
& \left|\frac{\left(P^{p} Q^{p} z \mathscr{D}_{P, Q} f(z) / F_{p} f(z)\right)+\alpha\left(P^{2 p+1} Q^{2 p+1} z^{2} \mathscr{D}_{P, Q}^{2} f(z) / F_{p} F_{p+1} f(z)\right)+1-\alpha}{(1-\alpha) L+M\left(\left(P^{p} Q^{p} z \mathscr{D}_{P, Q} f(z) / F_{p} f(z)\right)+\alpha\left(P^{2 p+1} Q^{2 p+1} z^{2} \mathscr{D}_{P, Q}^{2} f(z) / F_{p} F_{p+1} f(z)\right)\right)}\right| \\
& =\left|\frac{F_{p+1} P^{p} Q^{p} z \mathscr{D}_{P, Q} f(z)+\alpha P^{2 p+1} Q^{2 p+1} z^{2} \mathscr{D}_{P, Q}^{2} f(z)+(1-\alpha) F_{p} F_{p+1} f(z)}{L(1-\alpha) F_{p} F_{p+1} f(z)+M\left(F_{p+1} P^{p} Q^{p} z \mathscr{D}_{P, Q} f(z)+\alpha P^{2 p+1} Q^{2 p+1} z^{2} \mathscr{D}_{P, Q}^{2} f(z)\right)}\right| .
\end{aligned}
$$

Now, with the help of (4)-(6), we get, after some sim- for simplicity; then, (17) is equal to plification and using the notations,

$$
\begin{aligned}
\Psi_{1}(n)= & (1-\alpha) F_{p} F_{p+1}+P^{p} Q^{p} F_{p} F_{p+n} \\
& +\alpha P^{2 p+1} Q^{2 p+1} F_{p+n} F_{p+n-1}, \\
\Psi_{2}(n)= & L(1-\alpha) F_{p} F_{p+1}+M P^{p} Q^{p} F_{p} F_{p+n} \\
& +M \alpha P^{2 p+1} Q^{2 p+1} F_{p+n} F_{p+n-1},
\end{aligned}
$$

$$
\begin{aligned}
& \left|\frac{\sum_{n=1}^{\infty} \Psi_{1}(n) a_{p+n} z^{p+n}}{\left((1-\alpha)(L-M) F_{p} F_{p+1} / z^{p}\right)+\sum_{n=1}^{\infty} \Psi_{2}(n) a_{p+n} z^{p+n}}\right| \\
& =\left|\frac{\sum_{n=1}^{\infty} \Psi_{1}(n) a_{n+p} z^{n+2 p}}{(L-M)(1-\alpha) F_{p} F_{p+1}+\sum_{n=1}^{\infty} \Psi_{2}(n) a_{n+p} z^{n+2 p} \mid}\right| \\
& \leq \frac{\left|\sum_{n=1}^{\infty} \Psi_{1}(n) a_{n+p} z^{n+2 p}\right|}{\left|(L-M)(1-\alpha) F_{p} F_{p+1}\right|-\left|\sum_{n=1}^{\infty} \Psi_{2}(n) a_{n+p} z^{n+2 p}\right|} \\
& =\frac{\sum_{n=1}^{\infty} \Psi_{1}(n)\left|a_{n+p}\right||z|^{n+2 p}}{(L-M)(1-\alpha) F_{p} F_{p+1}-\left.\left|\sum_{n=1}^{\infty} \Psi_{2}(n)\right| a_{n+p}|| z\right|^{n+2 p} \mid} .
\end{aligned}
$$

As $|z|<1$, hence, the above is

$$
\begin{aligned}
& \leq \frac{\sum_{n=1}^{\infty} \Psi_{1}(n)\left|a_{n+p}\right|}{(L-M)(1-\alpha) F_{p} F_{p+1}-\sum_{n=1}^{\infty} \Psi_{2}(n)\left|a_{n+p}\right|} \\
& <1
\end{aligned}
$$

where we use inequality (16), and this completes the direct part.
Conversely, let $f \in \mathscr{M}_{P, Q}(p, \alpha, L, M)$ be given by (6). Then, from (12), for $z \in \mathbb{D}$, consider 


$$
\begin{aligned}
& \left|\frac{\left(P^{p} Q^{p} z \mathscr{D}_{P, Q} f(z) / F_{p} f(z)\right)+\alpha\left(P^{2 p+1} Q^{2 p+1} z^{2} \mathscr{D}_{P, Q}^{2} f(z) / F_{p} F_{p+1} f(z)\right)+1-\alpha}{(1-\alpha) L+M\left(\left(P^{p} Q^{p} z \mathscr{D}_{P, Q} f(z) / F_{p} f(z)\right)+\alpha\left(P^{2 p+1} Q^{2 p+1} z^{2} \mathscr{D}_{P, Q}^{2} f(z) / F_{p} F_{p+1} f(z)\right)\right)}\right| \\
& =\left|\frac{\sum_{n=1}^{\infty} \Psi_{1}(n) a_{n+p} z^{n+2 p}}{(L-M)(1-\alpha) F_{p} F_{p+1}+\sum_{n=1}^{\infty} \Psi_{2}(n) a_{n+p} z^{n+2 p}}\right|
\end{aligned}
$$

Since $|\Re \mathrm{e} z| \leq|z|$, we have

$$
\begin{aligned}
& \operatorname{Re}\left\{\frac{\sum_{n=1}^{\infty} \Psi_{1}(n) a_{n+p} z^{n+2 p}}{(L-M)(1-\alpha) F_{p} F_{p+1}+\sum_{n=1}^{\infty} \Psi_{2}(n) a_{n+p} z^{n+2 p}}\right\} \\
& \leq\left|\frac{\left(P^{p} Q^{p} z \mathscr{D}_{P, Q} f(z) / F_{p} f(z)\right)+\alpha\left(P^{2 p+1} Q^{2 p+1} z^{2} \mathscr{D}_{P, Q}^{2} f(z) / F_{p} F_{p+1} f(z)\right)+1-\alpha}{(1-\alpha) L+M\left(\left(P^{p} Q^{p} z \mathscr{D}_{P, Q} f(z) / F_{p} f(z)\right)+\alpha\left(P^{2 p+1} Q^{2 p+1} z^{2} \mathscr{D}_{P, Q}^{2} f(z) / F_{p} F_{p+1} f(z)\right)\right)}\right| \\
& <1 .
\end{aligned}
$$

Now, choose values of $z$ on the real axis so that

$$
\frac{1}{1-\alpha}\left(\frac{P^{p} Q^{p} z \mathscr{D}_{P, Q} f(z)}{F_{p} f(z)}+\alpha \frac{P^{2 p+1} Q^{2 p+1} z^{2} \mathscr{D}_{P, Q}^{2} f(z)}{F_{p} F_{p+1} f(z)}\right)
$$

is real. Upon clearing the denominator in (23) and letting $z \longrightarrow 1^{-}$through real values, we obtain (16).

\section{Coefficient Estimates}

In this section, we estimate the upper bounds of the coefficients of the functions in their series representation for this class.

Theorem 2. Let $f$ belong to $\mathscr{M}_{P, Q}(p, \alpha, L, M)$ and be ofform (6). Then,

$$
\begin{aligned}
&\left|a_{p+1}\right| \leq \frac{F_{p} F_{p+1}(1-\alpha)(L-M)}{\Psi_{1}(1)}, \\
&\left|a_{p+2}\right| \leq \frac{F_{p} F_{p+1}(1-\alpha)(L-M)}{\Psi_{1}(2)}+\frac{\left(F_{p} F_{p+1}(1-\alpha)(L-M)\right)^{2}}{\Psi_{1}(2) \Psi_{1}(1)}, \\
&\left|a_{p+3}\right| \leq \frac{F_{p} F_{p+1}(1-\alpha)(L-M)}{\Psi_{1}(3)}+\frac{\left(F_{p} F_{p+1}(1-\alpha)(L-M)\right)^{2}}{\Psi_{1}(3) \Psi_{1}(1)} \\
&+\frac{\left(F_{p} F_{p+1}(1-\alpha)(L-M)\right)^{2}}{\Psi_{1}(3) \Psi_{1}(2)}+\frac{\left(F_{p} F_{p+1}(1-\alpha)(L-M)\right)^{3}}{\Psi_{1}(3) \Psi_{1}(2) \Psi_{1}(1)}, \\
& h(z)=\frac{-1}{1-\alpha}\left(\frac{P^{p} Q^{p} z \mathscr{D}_{P, Q} f(z)}{F_{p} f(z)}+\alpha \frac{P^{2 p+1} Q^{2 p+1} z^{2} \mathscr{D}_{P, Q}^{2} f(z)}{F_{p} F_{p+1} f(z)}\right),
\end{aligned}
$$

where $\Psi_{1}(n)$ is defined in (18).

Proof. For $f \in \mathfrak{A}_{p}$, it will belong to $\mathscr{M}_{P, Q}(p, \alpha, L, M)$ if the following holds true:

$$
\frac{-1}{1-\alpha}\left(\frac{P^{p} Q^{p} z \mathscr{D}_{p, Q} f(z)}{F_{p} f(z)}+\alpha \frac{P^{2 p+1} Q^{2 p+1} z^{2} \mathscr{D}_{P, Q}^{2} f(z)}{F_{p} F_{p+1} f(z)}\right)<\frac{1+L z}{1+M z} .
$$

Now, if then

$$
h(z) \prec \frac{1+L z}{1+M z},
$$

and it will be of the form 


$$
h(z)=1+\sum_{n=1}^{\infty} d_{n} z^{n}
$$

However,

$$
\frac{1+L z}{1+M z}=1+(L-M) z+\cdots .
$$

Then, by Lemma 1, we obtain

$$
\left|d_{n}\right| \leq L-M
$$

From (27), we can easily obtain

$$
\begin{aligned}
& \frac{-1}{1-\alpha}\left(F_{p+1} P^{p} Q^{p} z \mathscr{D}_{P, Q} f(z)+\alpha P^{2 p+1} Q^{2 p+1} z^{2} \mathscr{D}_{P, Q}^{2} f(z)\right) \\
& =F_{p} F_{p+1} h(z) f(z) .
\end{aligned}
$$

Now, putting the series expansions of $h(z)$ and $f(z)$ in this and simplifying and comparing the coefficients of $z^{p+n}$ on both sides of the above equation, then

$$
\begin{aligned}
& -\frac{F_{p+n}\left(F_{p+1} P^{p} Q^{p}+\alpha P^{2 p+1} Q^{2 p+1} F_{p+n-1}\right)}{1-\alpha} a_{p+n} \\
& =F_{p} F_{p+1} a_{p+n}+F_{p} F_{p+1}\left(a_{p+n-1} d_{1}+a_{p+n-2} d_{2}+\cdots+a_{p+1} d_{n-1}\right),
\end{aligned}
$$

which implies that

$$
\begin{aligned}
& -\frac{F_{p+n}\left(F_{p+1} P^{p} Q^{p}+\alpha P^{2 p+1} Q^{2 p+1} F_{p+n-1}\right)+(1-\alpha) F_{p} F_{p+1}}{1-\alpha} a_{p+n} \\
& =F_{p} F_{p+1}\left(a_{p+n-1} d_{1}+a_{p+n-2} d_{2}+\cdots+a_{p+1} d_{n-1}\right) .
\end{aligned}
$$

Taking absolute on both sides,

$$
\left|-\frac{F_{p+n}\left(F_{p+1} P^{p} Q^{p}+\alpha P^{2 p+1} Q^{2 p+1} F_{p+n-1}\right)+(1-\alpha) F_{p} F_{p+1}}{1-\alpha} a_{p+n}\right|
$$$$
=\left|F_{p} F_{p+1}\left(a_{p+n-1} d_{1}+a_{p+n-2} d_{2}+\cdots+a_{p+1} d_{n-1}\right)\right| .
$$

Using the triangle inequality,

$$
\begin{aligned}
& \frac{F_{p+n}\left(F_{p+1} P^{p} Q^{p}+\alpha P^{2 p+1} Q^{2 p+1} F_{p+n-1}\right)+(1-\alpha) F_{p} F_{p+1}}{1-\alpha}\left|a_{p+n}\right| \\
& \leq F_{p} F_{p+1}\left(\left|a_{p+n-1}\right|\left|d_{1}\right|+\left|a_{p+n-2}\right|\left|d_{2}\right|+\cdots+\left|a_{p+1}\right|\left|d_{n-1}\right|\right) .
\end{aligned}
$$

Now, using (31), we obtain

$$
\begin{aligned}
& \frac{F_{p+n}\left(F_{p+1} P^{p} Q^{p}+\alpha P^{2 p+1} Q^{2 p+1} F_{p+n-1}\right)+(1-\alpha) F_{p} F_{p+1}}{1-\alpha}\left|a_{p+n}\right| \\
& \leq F_{p} F_{p+1}(L-M)\left(1+\left|a_{p+1}\right|+\left|a_{p+2}\right|+\cdots+\left|a_{p+n-1}\right|\right),
\end{aligned}
$$

which implies that

$$
\begin{aligned}
\left|a_{p+n}\right| & \leq \frac{(1-\alpha) F_{p} F_{p+1}(L-M)}{F_{p+n}\left(F_{p+1} P^{p} Q^{p}+a P^{2 p+1} Q^{2 p+1} F_{p+n-1}\right)+(1-\alpha) F_{p} F_{p+1}} \\
& \times\left(1+\left|a_{p+1}\right|+\left|a_{p+2}\right|+\cdots+\left|a_{p+n-1}\right|\right) .
\end{aligned}
$$

Now, by putting $n=1,2$, and 3 and using notation (18) for simplicity, we get the required result.

\section{Distortion and Growth-Type Inequalities}

Here, we discuss distortion-type inequality first. As in $(P, Q)$-analogue, we use $(P, Q)$-derivative in the distortion result instead of simple derivative. Furthermore, we prove the growth theorem for this class.

Theorem 3. If $f \in \mathscr{M}_{P, Q}(p, \alpha, L, M)$ and is of form (6), then, for $|z|=r$,

$$
\frac{F_{p}}{Q^{p} P^{p} r^{p+1}}-\mathscr{J}_{1} r^{p} \leq\left|\mathscr{D}_{P, Q} f(z)\right| \leq \frac{F_{p}}{Q^{p} P^{p} r^{p+1}}+\mathscr{J}_{1} r^{p},
$$

where

$$
\mathscr{F}_{1}=\frac{(1-\alpha)(L-M) F_{p} F_{p+1}}{(1-\alpha)(1+L) F_{p}+(1+M)\left(P^{p} Q^{p} F_{p+1}+\alpha P^{2 p+1} Q^{2 p+1} F_{p}\right)} .
$$

Proof. From (6) with the help of (4) and (5), we can write

$$
\begin{aligned}
\mathscr{D}_{P, Q} f(z)= & -\frac{F_{p}}{Q^{p} P^{p} z^{p+1}}+\sum_{n=1}^{\infty} F_{p+n} a_{n+p} z^{n+p-1}, \\
\Longrightarrow & \left|\mathscr{D}_{P, Q} f(z)\right| \leq \frac{F_{p}}{Q^{p} P^{p}|z|^{p+1}} \\
& +\sum_{n=1}^{\infty} F_{p+n}\left|a_{n+p}\right||z|^{n+p-1} .
\end{aligned}
$$

Since $|z|=r<1$, so $r^{n+p-1} \leq r^{p}$, and hence, 


$$
\mathscr{D}_{P, \mathrm{Q}} f(z) \leq \frac{F_{p}}{\mathrm{Q}^{p} P^{p} r^{p+1}}+r^{p} \sum_{n=1}^{\infty} F_{p+n}\left|a_{n+p}\right| .
$$

Similarly,

$$
\mathscr{D}_{P, Q} f(z) \geq \frac{F_{p}}{Q^{p} P^{p} r^{p+1}}-r^{p} \sum_{n=1}^{\infty} F_{p+n}\left|a_{n+p}\right|
$$

Since we know from (16) that

$$
\begin{aligned}
& \sum_{n=1}^{\infty}\left(\frac{(1+L)(1-\alpha) F_{p} F_{p+1}}{F_{p+n}}+(1+M)\left(Q^{p} P^{p} F_{p+1}+\alpha P^{2 p+1} Q^{2 p+1} F_{p+n-1}\right)\right) \\
& F_{p+n}\left|a_{n+p}\right| \leq(1-\alpha)(L-M) F_{p} F_{p+1}
\end{aligned}
$$

and

$$
\begin{aligned}
& \left((1+L)(1-\alpha) F_{p}+(1+M)\left(Q^{p} P^{p} F_{p+1}+\alpha P^{2 p+1} Q^{2 p+1} F_{p}\right)\right) \sum_{n=1}^{\infty} F_{p+n}\left|a_{n+p}\right| \\
& \leq \sum_{n=1}^{\infty}\left(\frac{(1+L)(1-\alpha) F_{p} F_{p+1}}{F_{p+n}}+(1+M)\left(Q^{p} P^{p} F_{p+1}+\alpha P^{2 p+1} Q^{2 p+1} F_{p+n-1}\right)\right) F_{p+n}\left|a_{n+p}\right|
\end{aligned}
$$

hence, by transitivity, we obtain

$$
\begin{aligned}
& \left((1+L)(1-\alpha) F_{p}+\left(Q^{p} P^{p} F_{p+1}+\alpha P^{2 p+1} Q^{2 p+1} F_{p}\right)(1+M)\right) \sum_{n=1}^{\infty} F_{p+n}\left|a_{n+p}\right| \\
& \leq(L-M)(1-\alpha) F_{p} F_{p+1},
\end{aligned}
$$

which implies that

$$
\sum_{n=1}^{\infty} F_{p+n}\left|a_{n+p}\right| \leq \frac{(1-\alpha)(L-M) F_{p} F_{p+1}}{(1-\alpha)(1+L) F_{p}+(1+M)\left(Q^{p} P^{p} F_{p+1}+\alpha P^{2 p+1} Q^{2 p+1} F_{p}\right)} .
$$

Now, we put this inequality in (42) and (43),

$$
\mathscr{D}_{P, Q} f(z) \leq \frac{F_{p}}{Q^{p} P^{p} r^{p+1}}+r^{p} \frac{(1-\alpha)(L-M) F_{p} F_{p+1}}{(1-\alpha)(1+L) F_{p}+(1+M)\left(Q^{p} P^{p} F_{p+1}+\alpha P^{2 p+1} Q^{2 p+1} F_{p}\right)} .
$$

Similarly,

$$
\mathscr{D}_{P, Q} f(z) \geq \frac{F_{p}}{Q^{p} P^{p} r^{p+1}}-r^{p} \frac{(1-\alpha)(L-M) F_{p} F_{p+1}}{(1-\alpha)(1+L) F_{p}+(1+M)\left(Q^{p} P^{p} F_{p+1}+\alpha P^{2 p+1} Q^{2 p+1} F_{p}\right)},
$$


from which we obtain the required result. class.

In the next result, we discuss the growth theorem for this

Theorem 4. Let $f \in \mathscr{M}_{P, Q}(p, \alpha, L, M)$ and is of form (6). Then, for $|z|=r$,

$$
\frac{1}{r^{p}}-\mathscr{J}_{2} r^{p} \leq|f(z)| \leq \frac{1}{r^{p}}+\mathscr{J}_{2} r^{p},
$$

where

$$
\mathscr{f}_{2}=\frac{(1-\alpha)(L-M) F_{p}}{(1-\alpha)(1+L) F_{p}+(1+M)\left(P^{p} Q^{p} F_{p+1}+\alpha P^{2 p+1} Q^{2 p+1} F_{p}\right)} .
$$

Proof. Consider

$$
\begin{aligned}
|f(z)| & =\left|\frac{1}{z^{p}}+\sum_{n=1}^{\infty} a_{n+p} z^{n+p}\right| \\
& \leq \frac{1}{\left|z^{p}\right|}+\sum_{n=1}^{\infty}\left|a_{n+p}\right||z|^{n+p} \\
& =\frac{1}{r^{p}}+\sum_{n=1}^{\infty}\left|a_{n+p}\right| r^{n+p} .
\end{aligned}
$$

Similarly,

$$
|f(z)| \geq \frac{1}{r^{p}}-r^{p} \sum_{n=1}^{\infty}\left|a_{n+p}\right| .
$$

Since we know from (16) that

$$
|f(z)| \leq \frac{1}{r^{p}}+r^{p} \sum_{n=1}^{\infty}\left|a_{n+p}\right| .
$$

$$
\begin{aligned}
& \sum_{n=1}^{\infty}\left((1+L)(1-\alpha) F_{p} F_{p+1}+(1+M)\left(P^{p} Q^{p} F_{p+1} F_{p+n}+\alpha P^{2 p+1} Q^{2 p+1} F_{p+n-1} F_{p+n}\right)\right)\left|a_{n+p}\right| \\
& \leq(1-\alpha)(L-M) F_{p} F_{p+1}
\end{aligned}
$$

and

$$
\begin{aligned}
& \left((1+L)(1-\alpha) F_{p}+\left(P^{p} Q^{p} F_{p+1}+\alpha P^{2 p+1} Q^{2 p+1} F_{p}\right)(1+M)\right) F_{p+1} \sum_{n=1}^{\infty}\left|a_{n+p}\right| \\
& \leq \sum_{n=1}^{\infty}\left((1+L)(1-\alpha) F_{p} F_{p+1}+(1+M)\left(P^{p} Q^{p} F_{p+1} F_{p+n}+\alpha P^{2 p+1} Q^{2 p+1} F_{p+n-1} F_{p+n}\right)\right)\left|a_{n+p}\right|,
\end{aligned}
$$

hence

$$
\begin{aligned}
& \left((1+L)(1-\alpha) F_{p}+\left(P^{p} Q^{p} F_{p+1}+\alpha P^{2 p+1} Q^{2 p+1} F_{p}\right)(1+M)\right) F_{p+1} \sum_{n=1}^{\infty}\left|a_{n+p}\right| \\
& \leq(1-\alpha)(L-M) F_{p} F_{p+1},
\end{aligned}
$$


which gives

$$
\sum_{n=1}^{\infty}\left|a_{n+p}\right| \leq \frac{(1-\alpha)(L-M) F_{p}}{(1-\alpha)(1+L) F_{p}+(1+M)\left(P^{p} Q^{p} F_{p+1}+\alpha P^{2 p+1} Q^{2 p+1} F_{p}\right)} .
$$

Now, by putting this value in (53) and (54),

$$
|f(z)| \leq \frac{1}{r^{p}}+r^{p} \frac{(1-\alpha)(L-M) F_{p}}{(1-\alpha)(1+L) F_{p}+(1+M)\left(P^{p} Q^{p} F_{p+1}+\alpha P^{2 p+1} Q^{2 p+1} F_{p}\right)} .
$$

Similarly,

$$
|f(z)| \geq \frac{1}{r^{p}}-r^{p} \frac{(1-\alpha)(L-M) F_{p}}{(1-\alpha)(1+L) F_{p}+(1+M)\left(P^{p} Q^{p} F_{p+1}+\alpha P^{2 p+1} Q^{2 p+1} F_{p}\right)},
$$

and from this, we get the required result.

\section{Radii of Convexity and Starlikeness}

In this section, we find the region where these functions will behave like convex and starlike functions of some order $\sigma$.

$$
r_{1}=\left(\frac{p(p-\sigma)(1-\alpha)(1+L) F_{p} F_{p+1}+(1+M)\left(P^{p} Q^{p} F_{p+1} F_{p+n}+\alpha P^{2 p+1} Q^{2 p+1} F_{p+n} F_{p+n-1}\right)}{(p+n)(n+p+\sigma)(1-\alpha)(L-M) F_{p} F_{p+1}}\right)^{1 /(n+2 p)} .
$$

Proof. Let $f \in \mathscr{M}_{P, Q}(p, \alpha, L, M)$. To prove $f \in \mathscr{M C}_{p}(\sigma)$, we only need to show

$$
\sum_{n=1}^{\infty} \frac{(p+n)(n+p+\sigma)}{p(p-\sigma)}\left|a_{n+p}\right||z|^{n+2 p}<1 .
$$

$$
\left|\frac{\left(z f^{\prime \prime}(z) / f^{\prime}(z)\right)+p+1}{\left(z f^{\prime \prime}(z) / f^{\prime}(z)\right)+1+2 \sigma-p}\right|<1
$$

Using (6) along with some simple computation yields

$$
\begin{aligned}
& \sum_{n=1}^{\infty}\left((1+L)(1-\alpha) F_{p} F_{p+1}+(1+M)\left(P^{p} Q^{p} F_{p+1} F_{p+n}+\alpha P^{2 p+1} Q^{2 p+1} F_{p+n} F_{p+n-1}\right)\right)\left|a_{n+p}\right| \\
& \leq(1-\alpha)(L-M) F_{p} F_{p+1} \\
& \Longrightarrow \sum_{n=1}^{\infty} \frac{(1+L)(1-\alpha) F_{p} F_{p+1}+(1+M)\left(P^{p} Q^{p} F_{p+1} F_{p+n}+\alpha P^{2 p+1} Q^{2 p+1} F_{p+n} F_{p+n-1}\right)}{(1-\alpha)(L-M) F_{p} F_{p+1}}\left|a_{n+p}\right|<1 .
\end{aligned}
$$


Now, inequality (63) will be true if the following holds

$$
\begin{aligned}
& \sum_{n=1}^{\infty} \frac{(p+n)(n+p+\sigma)}{p(p-\sigma)}\left|a_{n+p}\right||z|^{n+2 p} \\
& <\sum_{n=1}^{\infty} \frac{(1+L)(1-\alpha) F_{p} F_{p+1}+(1+M)\left(P^{p} Q^{p} F_{p+1} F_{p+n}+\alpha P^{2 p+1} Q^{2 p+1} F_{p+n} F_{p+n-1}\right)}{(1-\alpha)(L-M) F_{p} F_{p+1}}\left|a_{n+p}\right|
\end{aligned}
$$

which implies that

$$
\begin{aligned}
& \frac{(p+n)(n+p+\sigma)}{p(p-\sigma)}|z|^{n+2 p} \\
& \leq \frac{(1+L)(1-\alpha) F_{p} F_{p+1}+(1+M)\left(P^{p} Q^{p} F_{p+1} F_{p+n}+\alpha P^{2 p+1} Q^{2 p+1} F_{p+n} F_{p+n-1}\right)}{(1-\alpha)(L-M) F_{p} F_{p+1}},
\end{aligned}
$$

so

$$
|z|^{n+2 p}<\frac{p(p-\sigma)(1-\alpha)(1+L) F_{p} F_{p+1}+(1+M)\left(P^{p} Q^{p} F_{p+1} F_{p+n}+\alpha P^{2 p+1} Q^{2 p+1} F_{p+n} F_{p+n-1}\right)}{(p+n)(n+p+\sigma)(1-\alpha)(L-M) F_{p} F_{p+1}},
$$

from which we obtain

$$
\begin{aligned}
|z| & <\left(\frac{p(p-\sigma)(1-\alpha)(1+L) F_{p} F_{p+1}+(1+M)\left(P^{p} Q^{p} F_{p+1} F_{p+n}+\alpha P^{2 p+1} Q^{2 p+1} F_{p+n} F_{p+n-1}\right)}{(p+n)(n+p+\sigma)(1-\alpha)(L-M) F_{p} F_{p+1}}\right)^{1 /(n+2 p)} \\
& =r_{1}
\end{aligned}
$$

and thus the required condition.

Theorem 6. Let $f \in \mathscr{M}_{P, Q}(p, \alpha, L, M)$; then, $f$ will be in the class $\mathscr{M} \mathcal{S}_{p}^{*}(\sigma)$ for $|z|<r_{2}$, where

$$
r_{2}=\left(\frac{(p-\sigma)(1+L)(1-\alpha) F_{p} F_{p+1}+(1+M)\left(P^{p} Q^{p} F_{p+1} F_{p+n}+\alpha P^{2 p+1} Q^{2 p+1} F_{p+n} F_{p+n-1}\right)}{(n+p+\sigma)(1-\alpha)(L-M) F_{p} F_{p+1}}\right)^{1 /(n+2 p)}
$$


Proof. We know that $f$ belongs to $\mathscr{M}_{p}^{*}(\sigma)$ if and only if

$$
\left|\frac{\left(z f^{\prime}(z) / f(z)\right)+p}{\left(z f^{\prime}(z) / f(z)\right)-(p-2 \sigma)}\right| \leq 1 \text {. }
$$

$$
\sum_{n=1}^{\infty}\left(\frac{n+p+\sigma}{p-\sigma}\right)\left|a_{n+p}\right||z|^{n+2 p}<1
$$

Using (6) and upon simplification yield

$$
\text { Now, from (16), we can easily obtain }
$$

$$
\Longrightarrow \sum_{n=1}^{\infty} \frac{(1+L)(1-\alpha) F_{p} F_{p+1}+(1+M)\left(P^{p} Q^{p} F_{p+1} F_{p+n}+\alpha P^{2 p+1} Q^{2 p+1} F_{p+n} F_{p+n-1}\right)}{(1-\alpha)(L-M) F_{p} F_{p+1}}\left|a_{n+p}\right|<1 .
$$

For inequality (71) to be true, it will be enough if

$$
\begin{aligned}
& \sum_{n=1}^{\infty}\left(\frac{n+p+\sigma}{p-\sigma}\right)\left|a_{n+p}\right||z|^{n+2 p} \\
& <\sum_{n=1}^{\infty} \frac{(1+L)(1-\alpha) F_{p} F_{p+1}+(1+M)\left(P^{p} Q^{p} F_{p+1} F_{p+n}+\alpha P^{2 p+1} Q^{2 p+1} F_{p+n} F_{p+n-1}\right)}{(1-\alpha)(L-M) F_{p} F_{p+1}}\left|a_{n+p}\right| .
\end{aligned}
$$

However, this implies that

$$
\begin{aligned}
& \left(\frac{n+p+\sigma}{p-\sigma}\right)|z|^{n+2 p} \\
& <\frac{(1+L)(1-\alpha) F_{p} F_{p+1}+(1+M)\left(P^{p} Q^{p} F_{p+1} F_{p+n}+\alpha P^{2 p+1} Q^{2 p+1} F_{p+n} F_{p+n-1}\right)}{(1-\alpha)(L-M) F_{p} F_{p+1}},
\end{aligned}
$$

which gives

$$
|z|^{n+2 p}<\frac{(p-\sigma)(1+L)(1-\alpha) F_{p} F_{p+1}+(1+M)\left(P^{p} Q^{p} F_{p+1} F_{p+n}+\alpha P^{2 p+1} Q^{2 p+1} F_{p+n} F_{p+n-1}\right)}{(n+p+\sigma)(1-\alpha)(L-M) F_{p} F_{p+1}}
$$

and hence,

$$
\begin{aligned}
|z| & <\left(\frac{(p-\sigma)(1+L)(1-\alpha) F_{p} F_{p+1}+(1+M)\left(P^{p} Q^{p} F_{p+1} F_{p+n}+\alpha P^{2 p+1} Q^{2 p+1} F_{p+n} F_{p+n-1}\right)}{(n+p+\sigma)(1-\alpha)(L-M) F_{p} F_{p+1}}\right)^{1 /(n+2 p)} \\
& =r_{2} .
\end{aligned}
$$


Thus, the required result is proved.

\section{Convex Combination}

Theorem 7. The class $\mathscr{M}_{P, Q}(p, \alpha, L, M)$ is closed under convex combination.

Proof. Let us consider that we have two functions,

$$
f_{i}(z)=\frac{1}{z^{p}}+\sum_{k=1}^{\infty} a_{k+p, i} z^{k+p}, \quad i=1,2, z \in \mathbb{D},
$$

to prove our result, and we will show that

$$
h(z)=\gamma f_{1}(z)+(1-\gamma) f_{2}(z), \quad 0 \leq \gamma \leq 1,
$$

is in the class $\mathscr{M}_{P, Q}(p, \alpha, L, M)$.

As

$$
h(z)=\frac{1}{z^{p}}+\sum_{k=1}^{\infty}\left[\gamma a_{k+p, 1}+(1-\gamma) a_{k+p, 2}\right] z^{n},
$$

if we consider

$$
\begin{aligned}
& \sum_{n=1}^{\infty}\left((1+L)(1-\alpha) F_{p} F_{p+1}+(1+M)\left(P^{p} Q^{p} F_{p+1} F_{p+n}+\alpha P^{2 p+1} Q^{2 p+1} F_{p+n} F_{p+n-1}\right)\right) \\
& \left|\gamma a_{k+p, 1}+(1-\gamma) a_{k+p, 2}\right| \leq \gamma \sum_{n=1}^{\infty}\left((1+L)(1-\alpha) F_{p} F_{p+1}+(1+M)\left(P^{p} Q^{p} F_{p+1} F_{p+n}+\alpha P^{2 p+1} Q^{2 p+1} F_{p+n} F_{p+n-1}\right)\right)\left|a_{k+p, 1}\right| \\
& \quad \quad+(1-\gamma) \sum_{n=1}^{\infty}\left((1+L)(1-\alpha) F_{p} F_{p+1}+(1+M)\left(P^{p} Q^{p} F_{p+1} F_{p+n}+\alpha P^{2 p+1} Q^{2 p+1} F_{p+n} F_{p+n-1}\right)\right)\left|a_{k+p, 2}\right|,
\end{aligned}
$$

by using Theorem 1 for both $f_{1}(z)$ and $f_{1}(z)$, we have

$$
\begin{aligned}
& \sum_{n=1}^{\infty}\left((1+L)(1-\alpha) F_{p} F_{p+1}+(1+M)\left(P^{p} Q^{p} F_{p+1} F_{p+n}+\alpha P^{2 p+1} Q^{2 p+1} F_{p+n} F_{p+n-1}\right)\right)\left|\gamma a_{k+p, 1}+(1-\gamma) a_{k+p, 2}\right| \\
& \leq \gamma(1-\alpha)(L-M) F_{p} F_{p+1}+(1-\gamma)(1-\alpha)(L-M) F_{p} F_{p+1} \\
& =(1-\alpha)(L-M) F_{p} F_{p+1},
\end{aligned}
$$

and hence, by Theorem $1, h(z) \in \mathscr{M}_{P, Q}(p, \alpha, L, M)$.

\section{Conclusion and Future Study}

In this article, the concepts of post-quantum calculus are used to define a subfamily of meromorphic multivalent functions in association with Janowski functions. Some interesting results relating to geometry of these functions are proved for this class. This class not only generalizes some well-known classes in classical GFT but also covers many classes in $q$-analogue. This combination of post-quantum Calculus and GFT is an innovative concept and will of course lead to many new ideas and applications in the fields of mathematics. Various classes in the field of GFT such as analytic functions and harmonic functions can be generalized using the ideas and methods presented in this article.

\section{Data Availability}

Data used to support the findings of this study are included within the article.

\section{Conflicts of Interest}

The authors declare that they have no conflicts of interest.

\section{Authors' Contributions}

All authors jointly worked on the results, and they have read and approved the final manuscript.

\section{Acknowledgments}

The third author was supported by UKM (Grant GUP-2019032).

\section{References}

[1] F. H. Jackson, "On q-definite integrals," The Quarterly Journal of Pure and Applied Mathematics, vol. 41, pp. 193-203, 1910.

[2] F. H. Jackson, "XI.-On $q$-Functions and a certain difference operator," Transactions of the Royal Society of Edinburgh, vol. 46, no. 2, pp. 253-281, 1909.

[3] H. M. Srivastava and D. Bansal, "Close-to-convexity of a certain family of q-Mittag-Leffler functions," Journal of Nonlinear and Variational Analysis.vol. 1, no. 1, pp. 61-69, 2017.

[4] I. Aldawish and M. Darus, "Starlikeness of $q$-differential operator involving quantum calculus," Korean Journal of Mathematics, vol. 22, no. 4, pp. 699-709, 2014.

[5] T. M. Seoudy and M. K. Aouf, "Coefficient estimates of new classes of q-starlike and $q$-convex functions of complex 
order," Journal of Mathematical Inequalities, vol. 10, no. 1, pp. 135-145, 2016.

[6] L. Fan, Z. G. Wang, S. Khan, S. Hussain, M. Naeem, and T. Mahmood, "Coefficient bounds for certain subclasses of q-starlike functions," Mathematics, vol. 7, p. 969, 2019.

[7] S. Mahmood and J. Sokół, "New subclass of analytic functions in conical domain associated with Ruscheweyh $q$-differential operator," Results in Mathematics, vol. 71, no. 4, pp. 1345-1357, 2017.

[8] L. Shi, M. G. Khan, and B. Ahmad, "Some geometric properties of a family of analytic functions involving a generalised q-operator," Symmetry, vol. 12, pp. 1-11, 2020.

[9] Z. G. Wang, S. Hussain, M. Naeem, T. Mahmood, and S. Khan, "A subclass of univalent functions associated with $q$-analogue of Choi-Saigo-Srivastava operator," Hacettepe Journal of Mathematics and Statistics, vol. 49, no. 4, pp. 1471-1479, 2020.

[10] M. Naeem, S. Hussain, F. M. Sakar, T. Mahmood, and A. Rasheed, "Subclasses of uniformly convex and starlike functions associated with Bessel functions," Turkish Journal of Mathematics, vol. 43, no. 5, pp. 2433-2443, 2019.

[11] T. Mahmood, M. Naeem, S. Hussain, S. Khan, and S. Altinkaya, "A subclass of analytic functions defined by using Mittag-Leffer functions," Honam Mathematical Journal, vol. 42, pp. 577-590, 2020.

[12] B. Ahmad, M. G. Khan, M. G. Khan, B. A. Frasin, M. K. Aouf, and T. Mashwani, "On $q$-analogue of meromorphic multivalent functions in lemniscate of Bernoulli domain," AIMS Mathematics, vol. 6, no. 4, pp. 3037-3052, 2021.

[13] M. Arif and B. Ahmad, "New subfamily of meromorphic multivalent starlike functions in circular domain involving $q$ differential operator," Mathematica Slovaca, vol. 68, no. 5, pp. 1049-1056, 2018.

[14] B. Ahmad and M. Arif, "On a generalized subclass of meromorphic $p$-valent close to convex functions in $q$-analogue," International Journal of Maps in Mathematics, vol. 2, no. 2, pp. 148-158, 2019.

[15] B. Ahmad and M. Arif, "New subfamily of meromorphic convex functions in circular domain involving q-operator," International Journal of Analysis and Applications, vol. 16, pp. 75-82, 2018.

[16] R. Chakrabarti and R. Jagannathan, “A $(p, q)$-oscillator realization of two-parameter quantum algebras," Journal of Physics A: Mathematical and General, vol. 24, no. 13, 1991.

[17] M. Wachs and D. White, " $p, q$-stirling numbers and set partition statistics," Journal of Combinatorial Theory, Series A, vol. 56, no. 1, pp. 27-46, 1991.

[18] M. G. Khan, B. Ahmad, B. A. Frasin, and J. Abdel, "On Janowski analytic $(p, q)$-starlike functions in symmetric circular domain," Journal of Function Spaces, vol. 2020, Article ID 4257907, 6 pages, 2020.

[19] S. Kavitha, N. E. Cho, and G. Murugusundaramoorthy, "On $(p, q)$-quantum calculus involving quasi-subordination," in Proceedings of the Trend in mathematics, Advance in Algebra and Analysis International Conference on Advance in Mathematical Sciences, pp. 215-223, December 2017, http://www. springer.com/series/4961.

[20] S. Mahmood, Q. Z. Ahmad, H. M. Srivastava, N. Khan, B. Khan, and M. Tahir, "A certain subclass of meromorphically $q$-starlike functions associated with the Janowski functions," Journal of Inequalities and Applications, vol. 88, 2019.

[21] R. M. Ali and V. Ravichandran, "Classes of meromorphic alpha-convex functions," Taiwanese Journal of Mathematics, vol. 14, pp. 1479-1490, 2010.
[22] W. C. Royster, "Meromorphic starlike multivalent functions," Transactions of the American Mathematical Society, vol. 107, no. 2, p. 300, 1963.

[23] Ch. Pommerenke, "On meromorphic starlike functions," Pacific Journal of Mathematics, vol. 13, pp. 221-235, 1961.

[24] W. Rogosinski, "On the coefficients of subordinate functions," Proceedings of the London Mathematical Society, vol. 48, no. 2, pp. 48-82, 1943. 